\title{
Platoon signal priority in connected-autonomous vehicle environments: algorithm development and testing
}

\author{
Andalib Shams ${ }^{\mathrm{a}}$, Milan Zlatkovic ${ }^{\mathrm{b}}$ \\ ${ }^{a}$ lowa State University, Ames, lowa, USA \\ ${ }^{b}$ University of Wyoming, Laramie, Wyoming, USA
}

\section{ARTICLE INFO}

DOI: 10.31075/PIS.65.04.01

Professional paper

Received: 29/09/2019

Accepted: 14/11/2019

Corresponding author:

ashams@iastate.edu

Keywordsi:

Connected and Autonomous Vehicles Signal Control

Vehicle Platoons Microsimulation

\begin{abstract}
As traffic congestion increases day by day, it becomes necessary to improve the existing roadway facilities to maintain satisfactory operational and safety performances. New vehicle technologies, such as Connected and Autonomous Vehicles (CAV) have a potential to significantly improve transportation systems. Using the advantages of CAVs, this study developed signalized intersection control strategy algorithm that optimize the operations of CAVs and allow signal priority for connected platoons. The algorithm was tested in VISSIM microsimulation using a real-world urban corridor. The tested scenarios include a 2040 Do-Nothing scenario, and CAV alternatives with $25 \%, 50 \%, 75 \%$ and $100 \%$ CAV penetration rate. The results show a significant reduction in intersection delays $(26 \%-38 \%)$ and travel times $(6 \%-20 \%)$, depending on the penetration rate, as well as significant improvements on the network-wide level. CAV penetration rates of $50 \%$ or more have a potential to significantly improve all operational measures of effectiveness.
\end{abstract}

\section{INTRODUCTION}

Due to increased travel demand, traffic congestion has become a serious problem around the world. According to INRIX, in 2016 the USA lost $\$ 295$ billion due to congestion [1]. Inrix also predicted that the cumulative cost from 2013 to 2030 will be $\$ 2.8$ trillion). The improvement in existing vehicular and the introduction of new technologies has been rather significant in recent years. Even so, the current vehicular technology is only a fraction of what is being expected in the future [2]. It is expected that vehicles will be able to sense its environment and navigate the surroundings without any sort of human input. Furthermore, vehicles will be able to communicate with other vehicles, infrastructure, pedestrians or even with the cloud. Over the coming decades, improved technology will have a significant impact on traffic operations, safety and mobility [3]. These vehicles are introduced as Connected and Autonomous Vehicles (CAV). The U.S. Department of Transportation's National Highway Traffic Safety Administration (NHTSA) defined autonomous vehicles as "those in which operation of the vehicle occurs without direct driver input to control the steering, acceleration, and braking and are designed so that the driver is not expected to constantly monitor the roadway while operating in self-driving mode" [4]. At present, vehicles are already being deployed with some autonomous functionality, but the behavior of a fully autonomous car is yet to be explored. It is obvious that different car manufacturer will apply different logic to model the behavior of autonomous vehicles. But it is safe to say that these vehicles would have shorter headways, faster perception-reaction time and more uniform speeds than the conventional vehicles [5]. Most likely, autonomous vehicles will also possess the quality of connected vehicles. Connected vehicles will be able to communicate with other vehicles (Vehicle to Vehicle $\mathrm{V} 2 \mathrm{~V}$ ) as well as infrastructures (Vehicle to Infrastructure - V2I), like signal controllers. With the advantage of communication, connected vehicles will have different behavior than conventional vehicles. For example, these vehicles will be able to form platoons of vehicles and optimize their speed profile and routing decisions [6]. Though it is known that these vehicles will act more cooperatively than conventional vehicles, there is little development in the improvement of driving behaviors or intersection control strategies to make them more cooperative. 
Driver behavior models, such as the Intelligent Driver Model (IDM), were originally developed for the Adaptive Cruise Control (ACC) equipped vehicles. But IDM or modified versions of IDM are appropriate to model driver behavior of CAVs, because these models exclude the effect of human-reaction time [7]. In low CAV penetration rates, it will be difficult to maintain a platoon in the urban network due to different route choices and signalized intersections.

One of the challenges of improving driving behaviors or control strategies is the lack of testing platforms. Microscopic simulation can model the driving behavior of individual vehicles and their interaction with other vehicles. Also, simulation software VISSIM provides the opportunity to develop and test different algorithms, so it is appropriate for developing and testing driving behavior models and control strategies for CAV.

The study presented in this paper developed a signalized intersection control strategy algorithm and tested the performance of the Intelligent Driver Model, platooning, speed control, along with the signal control algorithm using VISSIM microsimulation. The signal control algorithm includes a platoon priority strategy, which works similarly to conventional signal priority by extending the green time for an approaching platoon under certain conditions. Although over time the penetration rate of CAVs will increase, initially they will operate in the mixed environment. CAV's interaction with the conventional vehicles will be complex. Understanding this necessity, the study analyzed CAV's impact on both connected and mixed environment, as well as their interaction with conventional vehicles.

\section{LITERATURE REVIEW}

Connected vehicles use wireless technology to establish a vehicle to vehicle (V2V) or vehicle to infrastructure (V2I) connection to share real-time information, which has the potential to increase traffic safety and mobility. Wireless technologies like Dedicated Short Range Communication (DSRC), Wi$\mathrm{Fi}$, and Bluetooth can be used to create a connected environment. DSRC is mostly similar to Wi-Fi communications, but $\mathrm{Wi}-\mathrm{Fi}$ is mainly for wireless local area network, whereas DSRC is highly secure and high-speed wireless communications. $75 \mathrm{MHz}$ of bandwidth at $5.9 \mathrm{GHz}$ was dedicated for DSRC whereas Wi-Fi mostly uses $2.4 \mathrm{GHz}$ and $5.8 \mathrm{GHz}$ radio bands. The $2.4 \mathrm{GHz}$ can be disrupted due to the other devices in the same area [8]. Another advantage of DSRC is the low latency of 0.02 second which means it takes 0.02 second in opening or closing a connection. DSRC is reserved for safety purposes in the connected environment, while the wireless technologies for non-safety application are yet to be fixed [3].
In the recent past, the U.S. DOT funded the deployment of CV prototypes in both test bed facilities and in the real world. According to the GSM Association, by 2020 nearly every new vehicle in the US will have a cellular-based telematics system. Very soon, a majority of the vehicles on U.S. roads will be equipped with CV technology [7]. Numerous current research efforts are dealing with different $\mathrm{CV}$ applications.

The U.S. Department of Transportation's National Highway Traffic Safety Administration (NHTSA) defined autonomous vehicles as those vehicles which can operate by itself without any human input. At present, most of the new vehicles have some autonomous functionality. With the progression of time vehicles will have more functions closer to fully autonomous vehicles. These vehicles will be integrated into the roadway through six-levels of driver assistance technology in coming years. At level 0 , the vehicle is totally human controlled, and at level 5 the vehicle will be fully automatic in all conditions [4]. Human-driven decision-making mechanisms are mostly obsolete for autonomous vehicles. Autonomous vehicles will have a lower Perception Reaction Time (PRT) than conventional vehicles and this low PRT leads to a different driving behavior than conventional vehicle's behavior [5].

PTV has provided a guideline on driving behaviors of Connected and Autonomous Vehicles [6]. CAVs will improve on conventional vehicles by:

- having smaller standstill distance

- following other vehicles with smaller oscillation distance

- accelerating faster and smoothly from standstill

- keeping constant speed with no or smaller oscillation at free flow

- forming platoons of vehicles

- adjusting speed profile to reach a green light at signals

- performing more co-operative lane changes, and smaller lateral distance to vehicles on adjacent lanes

Stanek et al. [5] and Atkins, Ltd. [9] measured the impact of autonomous vehicles based upon the PTV guidelines. Both these studies used the Wiedemann model for a congested network, which is a psychophysical traffic flow model. To make it realistic for autonomous vehicles, they set the parameter values based on low PRT. For example, Stanek et al. [5] reduced the minimum headway and safety distance reduction factor by $25 \%$. The results showed that in a congested network, if the CAV penetration rate is $10 \%$, the average delay will be reduced by $15 \%$, while for $100 \%$ penetration rate, the average delay will be reduced by $33 \%$. Atkins, Ltd. [9] measured the impact of $\mathrm{AV}$ on freeways. 
For $25 \%$ AV penetration rate, the average delay increased by $0.9 \%$, but for $100 \%$ penetration rate the average delay reduced by $33.8 \%$. Treiber et al. [10] developed the IDM for Autonomous Adaptive Cruise Control (ACC) systems. Later, Kesting et al. [11] developed Enhanced Intelligent Driver Model which is also suitable for Cooperative ACC. Both these driver models exclude human reaction time.

Milanés et al. [12] tested the IDM model in a field experiment under the free-flow conditions. Four production cars by Infiniti were equipped with Adaptive Cruise Control (ACC) controller, Cooperative ACC (CACC) controller, and original IDM model controller. This study found that although the IDM controller produced a smooth car-following model, it had a significant time gap in response to the speed change of the leader vehicle, ranging between $0.4 \mathrm{~s}$ and $0.7 \mathrm{~s}$. Therefore, they introduced a gap error and its derivative as a factor in deciding vehicle speed.

According to Fernades [13], grouping vehicles into platoons can effectively increase the roadway capacity and can be helpful in avoiding stop and go conditions in urban traffic. This study developed a method of keeping a lower headway in between platooned vehicles. Results from simulation experiments showed that this method reduced travel time when compared to a model with larger headways. Songchitruksa et al. [7] developed algorithms for platooning of CACCequipped vehicles with realistic lane changing behavior and realistic wireless reception. Results found that good wireless communication leads to more stable platoons, dedicated CACC lanes promote longer platoon formation, and higher market penetration of CACC results in a longer platoon.

Connected vehicles will be able to communicate with signal controllers wirelessly. Therefore, it is possible to adjust speed profiles to reach the beginning of a green signal. Jin et al. [14] developed a platoon-based multiagent intersection management system for connected vehicles. In this study, vehicles are allowed to form a platoon through V2V and V2I communication. Vehicle agent in the platoon communicates with the signal controllers to make a reservation based upon their arrival time at the intersection so that the vehicles plan their trajectory to meet the requirements (reservation, safety gap). Simulation of Urban Mobility (SUMO) software was used to build the traffic network, generate scenarios and evaluate the effectiveness of the proposed method. Results showed that the developed intersection management system reduced the average travel time by $12 \%$ to $30 \%$ compared to the traditional traffic control system, and $4 \%$ to $8 \%$ compared to the non-platoon based system. Feng et al. [15] presented a real-time adaptive phase allocation algorithm using connected vehicle's location and speed data. Phase sequence and duration are optimized through a bi-level optimization. The objective function was to minimize total delay and queue length.
To estimate the location and speed of unequipped vehicles, road segment near the intersection was divided into three regions: the queuing region, slowdown region, and free-flow region. Location and speed data of unequipped vehicles were determined through connected vehicle's data. Real-world model in VISSIM was used to test the performance. The total delay was reduced significantly compared to fully actuated control for high penetration rates, and comparable to actuated control for low penetration rates.

Islam and Hajbabaie [16] developed a distributed hierarchy-based coordinated network optimization of signal timing for a connected environment. The network signal timing optimization was reformulated from a centralized architecture to a decentralized approach and distributed mathematical programs were coordinated with each other to find the global optimality. Result showed that controlled queue length and maximized intersection throughput reduced travel times by $17 \%$ to $48 \%$ compared to actuated coordinated signals.

Until the conclusion of this study, research on impact assessment of CAVs within the simulation platform has been limited. Several studies tried to model AVs in VISSIM [5, 9]. Songchitruksa et al. [7] developed a simulation framework in VISSIM, but the developed model was tested for a freeway. The study presented in this paper developed a framework for the urban congested network. Also, potential impacts of CAVs on traffic operations are still not known. This study evaluated the developed framework to assess the impacts of CAVs.

\section{CAV SIGNAL PRIORITY ALGORITHM}

In near future, CAVs will bring transformative changes in the field of traffic operations, mobility, and safety [3]. Driving behavior of CAV will be more cooperative and will consider the overall benefits. Interaction with other vehicles will allow them to keep smaller headways with more uniform speed than the conventional vehicles [5]. These vehicles will also communicate with signal controllers, allowing for optimization of signal timing parameters. This section presents a methodology for platoon organization, speed control and signal optimization in the CAV environment. The methodology was implemented and tested in VISSIM microsimulation.

\subsection{Internal Model Development}

The internal driver model adapts the VISSIM's carfollowing (Wiedemann) model, lane change and vehicle speed parameters. The scope of the internal model is limited to autonomous vehicles. No V2V or V2I communication is possible through internal modeling. In this study, the lane changing behavior, lateral behavior, acceleration functions, and desired speeds are defined through the internal model. 


\subsection{External Model Development}

External model development was performed through VISSIM's Application Programming Interface (API), Component Object Modeling (COM) interface and an external driver model Dynamic Linked Library (DriverModel.dll). DriverModel.dll is appropriate for modeling continuous adjustment and for semiautomated or automated driving. COM interface is appropriate for modeling event-based processes and continuous adjustment. In this study, the driving behavior of CAV is modeled through DriverModel.dll, and the intersection control strategy algorithm is implemented through COM interface programming using Python 2.7.

\subsection{Car-Following Model}

The Wiedemann traffic flow model is a time-based, stochastic and microscopic car-following model, which is the default car-following model in VISSIM. It is a psychophysical model and appropriate for humandriven vehicles. To model Adaptive Cruise Control (ACC), researchers used Intelligent Driver Model (IDM). For Cooperative ACC (CACC) Enhanced IDM was used in previous studies. Both models exclude human reaction time, and therefore they are appropriate for modeling autonomous vehicles. Milanés et al. [12] updated the IDM equation to exclude the unrealistic negative values. This study uses this updated model as follows:

$$
\mathrm{a}_{\mathrm{IDM}}=\mathrm{a}\left[1-\left(\frac{\mathrm{v}}{\mathrm{v}_{0}}\right)^{\delta}-\left\{\frac{\mathrm{s}_{0}+\max \left(0, \mathrm{vT}+\frac{\mathrm{v} \Delta \mathrm{v}}{2 \sqrt{\mathrm{ab}}}\right)}{\mathrm{s}}\right\}\right]
$$

Here,

a - maximum acceleration - Maximum acceleration according to VISSIM's function

b $\quad$ - desired deceleration $\left(9 \mathrm{ft} / \mathrm{s}^{2}\right)$

$v_{0} \quad$ - desired speed in free-flow condition (posted speed limit)

v $\quad$ - current vehicle speed

s - bumper-to-bumper inter-vehicle clearance

so - vehicle to vehicle clearance in stand-still conditions (If the preceding vehicle is CAV then $3.3 \mathrm{ft}$, otherwise $4.9 \mathrm{ft}$ ).

$\mathrm{T} \quad$ - minimum steady state time gap $-1.1 \mathrm{~s}$ If the driver decides to brake for a yellow or red light, the vehicle will be assigned a constant deceleration value bapplied:

brequired - $v^{2} / 2 d x$

$b_{\text {applied }}$ - minimum (brequired, $\left.b_{\max }\right)$

Here,

$\mathrm{dx} \quad$ - distance to stop line

brequired - required deceleration

$b_{\max }$ - maximum deceleration according to VISSIM's function.
This driver model was coded into External Driver Model Dynamic Linked Library (DriverModel.dll) and called in each simulation time step $(0.1 \mathrm{~s})$.

\subsection{Lane Changing and Lateral Behavior}

Based on the guideline provided by PTV [6], and in the lack of other available data, Stanek et al. [5] assumed that the reduction of minimum headway and safety distance reduction factor would be $25 \%$. Maximum deceleration for cooperative braking was increased from $9.84 \mathrm{ft} / \mathrm{s}^{2}$ to $13.12 \mathrm{ft} / \mathrm{s}^{2}$, and the cooperative lane change parameter was selected to facilitate the lanechanging cooperatively. This study also used these parameter values.

\subsection{Formation of Vehicle Platoons}

It is expected that connected vehicles will form platoons of vehicles. According to Jin et al. [14], grouping vehicles into platoons will increase roadway capacity. For the purpose of this study, the authors developed a platoon-forming algorithm as shown in Figure 1.

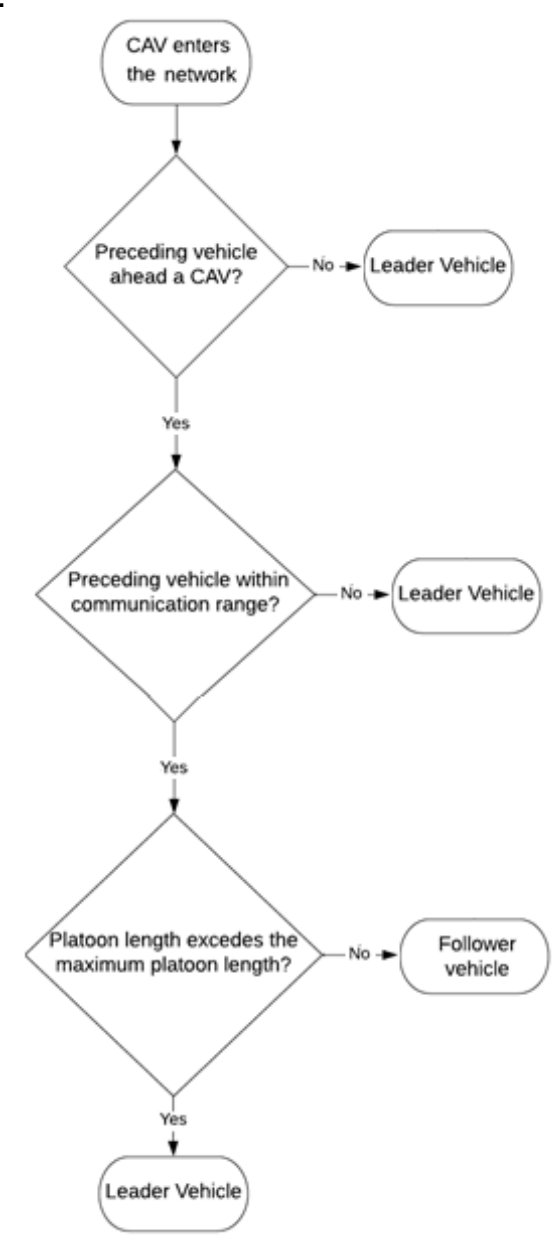

Figure 1: Platoon Formation Algorithm 
When a vehicle is released into the network, it will search for its preceding vehicle in the same lane. If the preceding vehicle is a connected vehicle and within the communication range, the new vehicle will request to join the platoon of the preceding vehicle. If the platoon already reached its maximum platoon length, the new vehicle will take the role of leader, otherwise a follower. If the preceding vehicle is not a connected vehicle, the newer vehicle will be the leader vehicle. For this study, it is assumed that the $\mathrm{V} 2 \mathrm{~V}$ communication range is $300 \mathrm{ft}$ and the maximum platoon length is five vehicles.

\subsection{Trajectory Planning}

Trajectory Planning is one of the integrated traffic signal control algorithms. Each CAV communicates with the signal controller to receive the information about the start time of the next green or red on its approaching signal phase. If the signal state is green, the signal controller will send information about the next red time. The vehicle will modify its desired speed so that it can pass the intersection before the start of the red signal. During a green signal, the desired speed of a CAV is derived from equations below. If the desired speed of a CAV is higher than the posted speed limit, the CAV will obey the posted speed limit. The CAV also maintains a safe distance with the adjacent vehicle.

Speed $_{\text {required }}=\frac{\text { Distance to signal }}{\text { Time until next red }}$

Speed $_{\text {desired }}=\min [\max ($ Speed required, Original Desired Speed), posted speed limit]

If the signal state is red, the signal controller will send the information about the start time of next green. The vehicle will change its desired speed so that it can reach the intersection at the beginning of the green signal without stopping. At a red signal, the desired speed will be derived from following rules:

Speed $_{\text {required }}=\frac{\text { Distance to signal }}{\text { Time until next green }}$

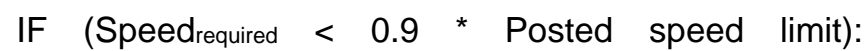
Speeddesired $=$ Original Desired Speed

\section{ELSE Speed $_{\text {desired }}=$ Speed $_{\text {required }}$}

The IF condition prevents unrealistically low speeds by keeping the minimum speed of the vehicles at $90 \%$ of the posted speed limit. The flow chart of the algorithm is shown in Figure 2.

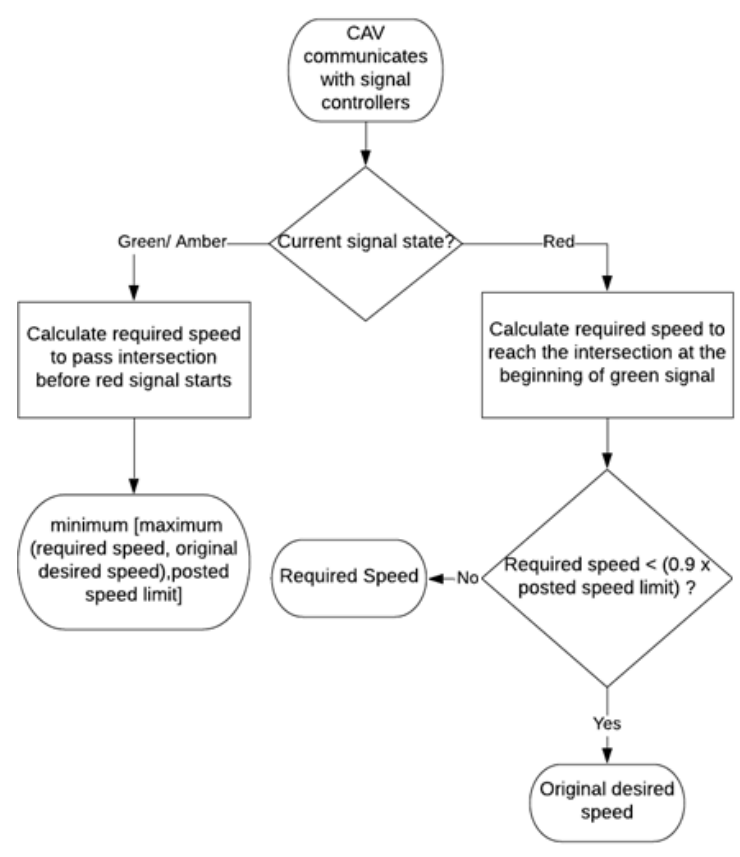

Figure 2. Desired Speed Selection

\subsection{Green Extension Time}

Green Extension Time is the second integrated traffic signal control algorithm. In a mixed environment, it is relatively difficult to form a platoon and maintain it. $A$ formed platoon can break due to different routes of vehicles or signal timing. This algorithm maintains a complete platoon by facilitating its movement through the signalized intersection. If the signal state is green and a platoon requests more time to pass the intersection, the signal controller will consider extending the green time. The maximum allowable green extension time in this study is 5 seconds, which is sufficient time for the entire platoon of maximum five vehicles to pass the stop bar based on the previously defined car following behavior. If the trajectory plan of the platoon leader suggests that the leader will reach the intersection after the regular green time, the signal controller will not allow green extension time. The flow chart of the algorithm is shown in Figure 3.

\section{STUDY NETWORK AND BASE MODEL DEVELOPMENT}

The algorithm was tested in microsimulation on a realworld network. The test-bed network for this study is a section of Redwood Road in Taylorsville, Utah, spanning two miles between $3500 \mathrm{~S}$ and $4700 \mathrm{~S}$, as shown in Figure 4. There are seven signalized intersections along this corridor. The base simulation model was created using a combination of VISUM (mesoscopic simulation with traffic assignment abilities) and VISSIM (microscopic simulation). The initial model of existing conditions was created in VISUM and calibrated for traffic volumes using the 
VISUM's built-in functions. The geometry of the model was created based on field conditions. Traffic volume and turning movement count (TMC) data were collected for the intersections of Redwood Road with $3500 \mathrm{~S}$ and $4700 \mathrm{~S}$, as the major intersections, using the Utah Department of Transportation (UDOT) Signal Performance Matrices (SPM) system. The data were collected for the PM peak period (4:00 PM -6:00 PM) for every 15 minutes with September 28, 2016 as the representative weekday. These turning movement count data were added to the VISUM model. Additional traffic volume data were obtained from UDOT's AADT maps and an existing Synchro model of the corridor. An initial Origin-Destination (OD) matrix was prepared and loaded into the model.

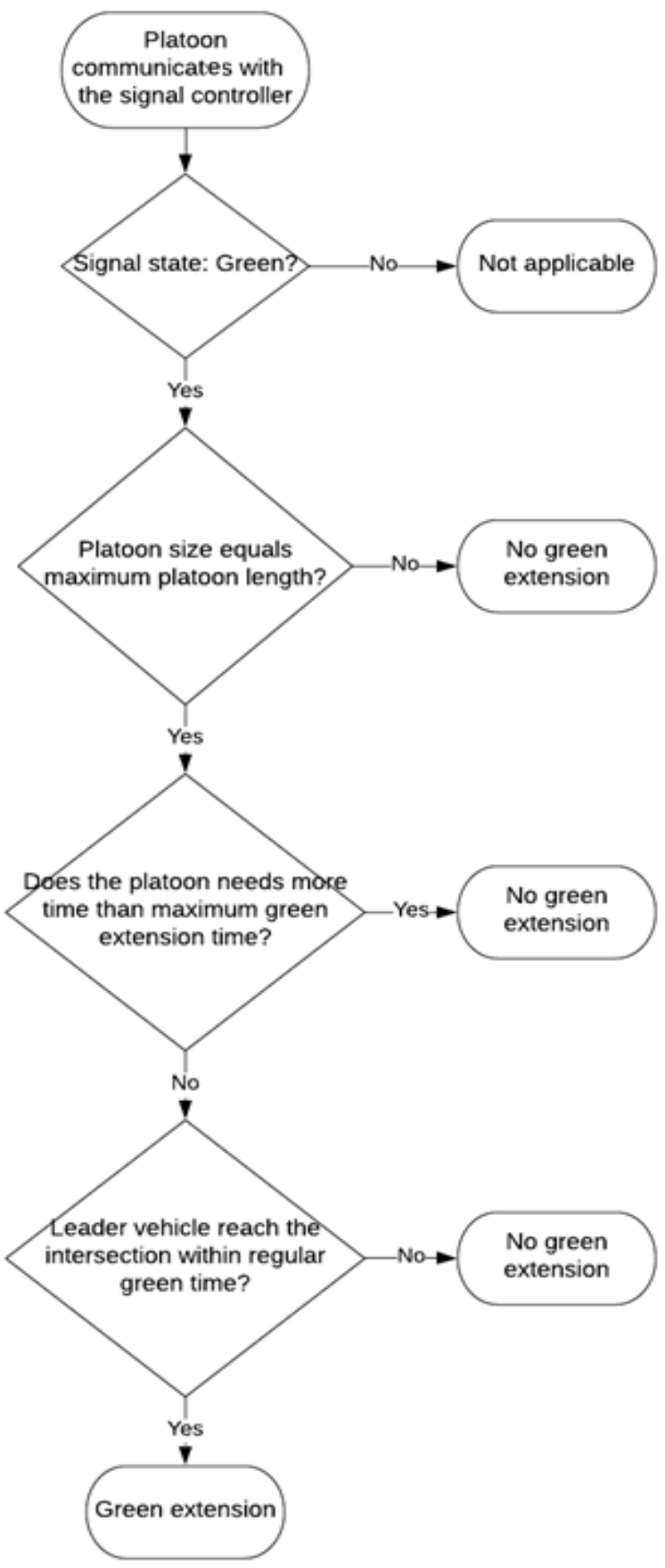

Figure 3. Green Extension Strategy

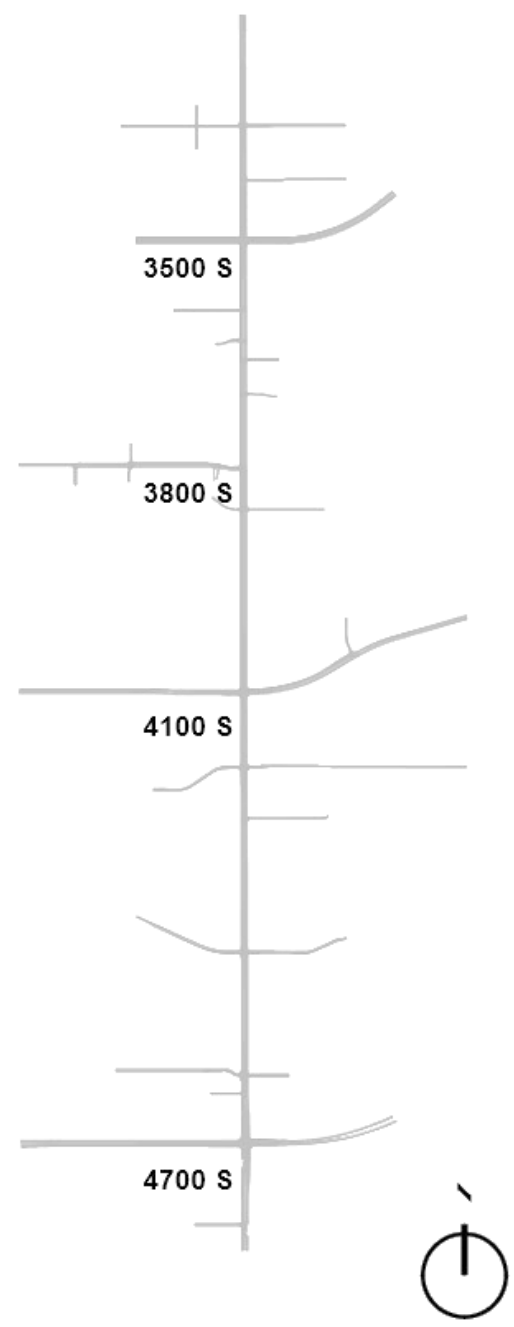

Figure 4: Study Network

The VISUM's T-Flow Fuzzy function was used to correct and calibrate the OD matrix to match the existing traffic volumes. After the volume calibration in VISUM, the network with traffic volume inputs and routing decisions was exported to VISSIM for further model development. The model was upgraded based on the actual intersection geometries provided by the VISSIM background maps. The geometry was checked through Google Street View and field visits to ensure that the model represents actual conditions. All signalized intersections were modeled according to the existing signal timings downloaded from the UDOT's MaxView software. Detector size and location were modeled according to the UDOT's standards and recommendations. The signal timing data also included the signals along the corridors parallel to Redwood Road. In the models, these signals were created as "dummy signals", since no volume data were available for those intersections. To maintain coordination and platooning, minimum phase recalls was coded for the "dummy signals" in VISSIM. 
The base model was calibrated for 15-minute intersection turning counts for the intersections of 3500 $S$ and $4700 \mathrm{~S}$, shown in Figure 5 . The $\mathrm{R}^{2}$ value of 0.96 shows a good match between the field and simulation data.

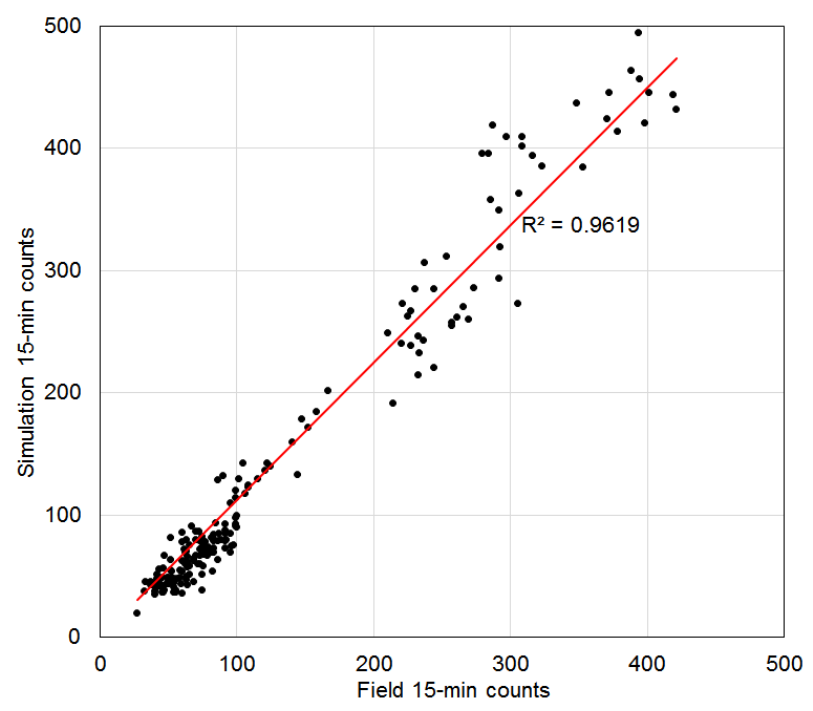

Figure 5: Base Model Calibration

\section{2040 MODELS AND ALTERNATIVE CAV SCENARIOS}

The algorithm testing was performed for the 2040 target year. For this purpose, first a Do-Nothing model was developed, using a projected travel demand for 2040 along this corridor. Traffic projections and volume balancing were used to create new demand for the VISSIM model. The network geometry did not change. The only parameters that changed were signal timing settings. Synchro was used to optimize signals for the new demand levels, and traffic control in VISSIM was updated based on this optimization. Four CAV alternative scenarios were modeled, with $25 \%, 50$, $75 \%$ and $100 \%$ CAV penetration rate. All CAV models were derived from the 2040 Do-Nothing model. The results from the five scenarios were used to assess the effectiveness of proposed algorithms.

\section{RESULTS AND DISCUSSION}

To evaluate the impacts of CAVs and the developed control programs, VISSIM was coded to record intersection performances, segment travel times, and overall network performances. Each scenario was run for five differentially seeded simulations (with the same sequence of random seeds among scenarios), where each simulation was two and a half hours long (30 minutes of warm-up time and two hours of output recording). The average values of the five simulations run for each scenario were used in the analysis. Twotailed paired t-test with a $95 \%$ confidence level was used to assess the statistical significance in the results from various scenarios.

\subsection{Intersection Performance}

Vehicle delays were measured for each signalized intersection along the analyzed corridor. The total weighted intersection delays obtained through simulations are provided in Table 1.

Table 1: Intersection Performance Comparison

\begin{tabular}{|c|c|c|c|c|c|}
\hline \multicolumn{6}{|c|}{ TOTAL INTERSECTION DELAY (hours) } \\
\hline Signal & $\begin{array}{l}\text { Do- } \\
\text { Nothing } \\
\text { (DN) }\end{array}$ & $\begin{array}{l}25 \% \\
\text { CAV }\end{array}$ & $\begin{array}{l}50 \% \\
\mathrm{CAV}\end{array}$ & $\begin{array}{l}75 \% \\
\text { CAV }\end{array}$ & $\begin{array}{c}100 \% \\
\text { CAV }\end{array}$ \\
\hline $3500 \mathrm{~S}$ & 467.7 & 267.7 & 246.7 & 237.4 & 231.3 \\
\hline $3800 \mathrm{~S}$ & 14.5 & 12.0 & 11.0 & 10.4 & 10.1 \\
\hline $4100 \mathrm{~S}$ & 320.5 & 198.9 & 183.6 & 178.4 & 172.6 \\
\hline $4200 \mathrm{~S}$ & 96.2 & 60.2 & 58.2 & 54.2 & 42.4 \\
\hline $4450 \mathrm{~S}$ & 125.2 & 115.9 & 95.9 & 82.5 & 71.4 \\
\hline $4610 \mathrm{~S}$ & 127.0 & 122.0 & 109.0 & 131.9 & 108.0 \\
\hline $4700 \mathrm{~S}$ & 272.0 & 269.5 & 283.5 & 254.4 & 244.3 \\
\hline Total & 1423.1 & 1046.5 & 988.2 & $949.7^{*}$ & $880.8^{*}$ \\
\hline $\begin{array}{c}\text { Compared } \\
\text { to DN }\end{array}$ & NA & $-26 \%$ & $-31 \%$ & $-33 \%$ & $-38 \%$ \\
\hline
\end{tabular}

Intersection performance result suggests that the total intersection delay during the 2-hour PM peak period reduced for any level of CAV penetration. For $25 \%$, $50 \%, 75 \%$ and $100 \%$ CAVs, the total intersection delays along the tested corridor reduced by $26 \%, 31 \%$, $33 \%$ and $38 \%$, respectively. Statistical tests show a statistically significant difference for CAV penetration rate of $75 \%$ or $100 \%$ compared to the Do-Nothing scenario.

\subsection{Segment Travel Times}

Travel times for vehicular traffic were measured for segments between each pair of signalized intersections. The average travel times obtained through simulations are given in Table 2 for different scenarios.

The reduction in travel times is statistically significant for all CAV penetration rates in both directions, except for the 25\% CAV in the northbound direction. The results also show a consistent reduction in travel times in both directions for the same CAV rate. The time reduction continuously increases from $6 \%$ for $25 \%$ CAVs, to $20 \%$ for $100 \%$ CAVs. 
Table 2: Segment Travel Times Comparison

\begin{tabular}{|c|c|c|c|c|c|}
\hline \multicolumn{6}{|c|}{ AVERAGE TRAVEL TIME (seconds) } \\
\hline Segment & $\begin{array}{c}\text { Do-Nothing } \\
\text { (DN) }\end{array}$ & $\begin{array}{l}25 \% \\
\text { CAV }\end{array}$ & $\begin{array}{l}50 \% \\
\text { CAV }\end{array}$ & $\begin{array}{l}75 \% \\
\text { CAV }\end{array}$ & $\begin{array}{l}100 \% \\
\text { CAV }\end{array}$ \\
\hline \multicolumn{6}{|l|}{ North Bound } \\
\hline $4700-4610$ & 70.9 & 57.2 & 53.2 & 47.6 & 44.8 \\
\hline $4610-4450$ & 44.7 & 41.4 & 38.1 & 37.4 & 29.8 \\
\hline $4450-4200$ & 57.1 & 56.7 & 53.7 & 52.7 & 50.1 \\
\hline $4200-4100$ & 54.9 & 53.4 & 50.4 & 49.2 & 46.1 \\
\hline $4100-3800$ & 48.4 & 47.6 & 46.6 & 45.8 & 43.8 \\
\hline $3800-3500$ & 91.4 & 89.7 & 89.2 & 83.5 & 77.7 \\
\hline Total & 367.4 & 346.0 & $331.2^{*}$ & $316.1^{*}$ & $292.4^{*}$ \\
\hline $\begin{array}{l}\text { Compared to } \\
\text { DN }\end{array}$ & NA & $-6 \%$ & $-10 \%$ & $-14 \%$ & $-20 \%$ \\
\hline \multicolumn{6}{|l|}{ South Bound } \\
\hline $3500-3800$ & 48.9 & 45.7 & 44.0 & 42.9 & 41.1 \\
\hline $3800-4100$ & 119.6 & 109.7 & 106.4 & 103.2 & 101.4 \\
\hline $4100-4200$ & 18.0 & 17.6 & 16.6 & 15.0 & 13.6 \\
\hline $4200-4450$ & 51.5 & 47.6 & 42.8 & 41.4 & 39.8 \\
\hline $4450-4600$ & 78.8 & 72.9 & 70.2 & 68.9 & 65.0 \\
\hline $4600-4700$ & 59.0 & 56.0 & 52.3 & 49.4 & 43.8 \\
\hline Total & 375.8 & $349.5^{\star}$ & $332.2^{*}$ & $320.7^{*}$ & $304.8^{*}$ \\
\hline $\begin{array}{l}\text { Compared to } \\
\text { DN }\end{array}$ & NA & $-7 \%$ & $-12 \%$ & $-15 \%$ & $-19 \%$ \\
\hline
\end{tabular}

\subsection{Network Performance}

The effects of the analyzed scenarios were further assessed on the overall network using the most relevant parameters, such as the average speed, total travel time, average stopped delay and a total number of stops. A summary of network performance results is presented in Table 3.

The overall network-wide performance was better for all CAV scenarios compared to Do-Nothing. For the $25 \%$ CAV penetration rate, the average speed reduced by $1.5 \%$ compared to the Do-Nothing scenario. This is due to the speed control algorithm and more interactions with non-CAV vehicles. Also, the platoon formation is harder to achieve due to the lower CAV percentage. The average speed increased for $50 \%$ or above CAV penetration rates. For $100 \%$ CAV the average speed increased by $8.1 \%$.

Other parameters for network performance evaluation also suggest an improvement in traffic operations with the increase in CAV rates. The total number of stops is an important parameter as it can measure the performance of the trajectory planning algorithm. For $25 \%$ CAV the number of stops increased, but with the increase in penetration rate, the number of stops decreased. For $100 \%$ CAVs the number of stops reduced by $36 \%$.

\section{CONCLUSIONS}

In the future, with the introduction of new vehicular technologies, vehicles will be able to communicate with other vehicles and the traffic environment. Their behavior will have significant impacts on all aspects of traffic operations and safety.

This paper describes the development and testing of a platoon signal priority algorithm that enables the connected vehicles to form platoons and facilitate their movement through signalized intersections. The algorithm was tested in VISSIM microsimulation, on a real-world corridor along Redwood Road in Taylorsville, Utah. Four scenarios with 25\%, 50, 75\% and $100 \%$ CAV penetration rates were used in the analysis.

Comparing the performance CAV scenarios to the 2040 Do-Nothing scenario, the following conclusions were reached:

- Total intersection delays and travel times decreased with an increase in CAV penetration rates.

- For $50 \%$ or more CAV penetration rate, the average speed continued to increase with the increase in penetration rate.

- If the CAV penetration rate was more than 50\%, the number of vehicular stops decreased continuously, suggesting significant benefits of the integrated trajectory planning algorithm.

- With $25 \%$ CAV rate, the number of vehicular stops increased, while the average speed decreased. This is due to the significant interaction between CAV and non-CAV vehicles. With lower penetration rates it is difficult to form platoons, therefore reducing the effectiveness of the green extension algorithm.

- The total delay and total travel time of the overall network decreased with the increase in penetration rate of CAV.

Future studies will keep on improving the algorithm, considering optimization for low CAV penetration rates, optimizations in lane changing behavior, as well as introducing a segment-based speed optimization that will apply to all vehicles (CAV and non-CAV) within each segment and direction. 
Table 3: Network Performance Comparison

\begin{tabular}{|c|c|c|c|c|c|c|c|c|c|}
\hline & \multirow{2}{*}{$\begin{array}{c}\begin{array}{c}\text { Do- } \\
\text { Nothing } \\
\text { (DN) }\end{array} \\
\text { Value }\end{array}$} & \multicolumn{2}{|c|}{$25 \%$ CAV } & \multicolumn{2}{|c|}{$50 \%$ CAV } & \multicolumn{2}{|c|}{$75 \%$ CAV } & \multicolumn{2}{|c|}{$100 \%$ CAV } \\
\hline & & Value & $\begin{array}{c}\text { Compared } \\
\text { to DN }\end{array}$ & Value & $\begin{array}{l}\text { Compared } \\
\text { to DN }\end{array}$ & Value & $\begin{array}{c}\text { Compared } \\
\text { to DN }\end{array}$ & Value & $\begin{array}{c}\text { Compared } \\
\text { to DN }\end{array}$ \\
\hline Average Speed (mph) & 13.6 & 13.4 & $-1.5 \%$ & 14.3 & $4.9 \%$ & 14.5 & $6.8 \%$ & 14.7 & $8.1 \%$ \\
\hline Total Travel Time (hr) & 3204.0 & 3052.8 & $-4.7 \%$ & 2869.9 & $-10.4 \%$ & 2859.0 & $-10.8 \%$ & 2848.5 & $-11.1 \%$ \\
\hline Total Delay (hr) & 2067.4 & 1977.9 & $-4.3 \%$ & 1780.9 & $-13.9 \%$ & 1756.1 & $-15.1 \%$ & 1735.1 & $-16.1 \%$ \\
\hline Number of Stops & 141289 & 159066 & $12.6 \%$ & 121378 & $-14.1 \%$ & 99486 & $-29.6 \%$ & 90800 & $-35.7 \%$ \\
\hline
\end{tabular}

\section{Acknowledgements}

The authors thank Avenue Consultants from Taylorsville, Utah, for their help with this study. Their input on modeling and analysis, as well as their help in obtaining the data, providing future demand levels and performing field visits, was invaluable for the successful completion of the work presented in this paper.

\section{References}

[1] Cookson, G., and Pishue, B. (2017). INRIX Global Traffic Scorecard. INRIX Research, http://inrix.com/scorecard/

[2] Autocaat (2018). Automated and Connected Vehicles. Autocaat.org,

http://autocaat.org/Technologies/Automated_and_Connected_ Vehicles/ (Accessed 07/19/2018).

[3] Hill, C., Hamilton, A., and Krueger, G. (2014.). Module 13: Connected Vehicles Introduction: Definition and Programmatic Overview. Intelligent Transportation systems, Joint Program Office (ITS https://www.pcb.its.dot.gov/eprimer/module13.aspx (Accessed 09/28/2019).

[4] National Highway Traffic Safety Administration (NHTSA) (2013). Automated Vehicles for Safety. https://www.nhtsa.gov/technology-innovation/automatedvehicles-safety (Accessed 09/28/2019)

[5] Stanek, D., Huang, E., Milam, R., and Wang, Y. (2018). Measuring Autonomous Vehicle Impacts on Congested Networks Using Simulation. Transportation Research Procedia, 728(408).

[6] PTV Group. (2017). PTV VISSIM and Connected-Autonomous Vehicles. http://www.sfbayite.org/wpcontent/uploads/2017/04/1\%20VISSIM_CAV_SFITE_April201 7.pdf (Accessed 09/28/2019)

[7] Songchitruksa, P., Bibeka, A., Lin, L., and Zhang, Y. (2016). Incorporating Driver Behaviors into Connected and Automated Vehicle Simulation. Advancing Transportation Leadership and Safety (ATLAS) Center, Report ATLAS-2016-13. http://www.atlas-center.org/wpcontent/uploads/2014/10/ATLAS-Research-ReportSongchitruksa-ATLAS-2016-13.pdf (Accessed 09/28/2019)
[8] Intelligent Transportation Systems Joint Program Office (ITSJPO) (2018). Connected Vehicles. https://www.its.dot.gov/cv_basics (Accessed 6/21/2019)

[9] Atkins, Ltd. (2016). Research on the Impacts of Connected and Autonomous Vehicles (CAVs) on Traffic Flow.

https://assets.publishing.service.gov.uk/government/uplo ads/system/uploads/attachment data/file/530091/impact s-of-connected-and-autonomous-vehicles-on-traffic-flowsummary-report.pdf (Accessed 07/20/2018)

[10] Treiber, M., Hennecke, A., and Helbing, D. (2000). Congested traffic states in empirical observations and microscopic simulations. Physical Review E - Statistical Physics, Plasmas, Fluids, and Related Interdisciplinary Topics, 62(2), 1805-1824.

[11] Kesting, A., Treiber, M., Schönhof, M., and Helbing, D. (2008). Adaptive cruise control design for active congestion avoidance. Transportation Research Part C: Emerging Technologies, 16(6), 668-683.

[12] Milanés, V., and Shladover, S. (2014). Modeling cooperative and autonomous adaptive cruise control dynamic responses using experimental data. Transportation Research Part C: Emerging Technologies, Vol. 48, 285-300.

[13] Fernandes, P., and Nunes, U. (2010). Platooning of autonomous vehicles with intervehicle communications in SUMO traffic simulator. IEEE Conference on Intelligent Transportation Systems, Proceedings, ITSC, 1313-1318.

[14] Jin, Q., Wu, G., Boriboonsomsin, K., Barth, M., and Member, S. (2013). Platoon - Based Multi - Agent Intersection Management for Connected Vehicle. 16th International IEEE Conference on Intelligent Transportation Systems (ITSC 2013), 1462-1467.

[15] Feng, Y., Head, L., Khoshmagham, S., and Zamanipour, M. (2015). A real-time adaptive signal control in a connected vehicle environment. Transportation Research Part C: Emerging Technologies, Vol. 55, 460473

[16]Islam, S. M. A. B. Al, and Hajbabaie, A. (2017). Distributed coordinated signal timing optimization in connected transportation networks. Transportation Research Part C: Emerging Technologies, Vol. 80, 272285. 